\title{
Symbolic behavior in regular classrooms: a specification of symbolic and non-symbolic behavior
}

\author{
Stefan Billinger ${ }^{*}$ and Torsten Norlander ${ }^{2}$ \\ ISchool of Health and Medical Sciences, Örebro University, Örebro, Sweden \\ 2 Department of Psychology, Karlstad University, Karlstad, Sweden
}

\section{Edited by:}

Ann X. Huang, Duquesne University, USA

\section{Reviewed by:}

Ann X. Huang, Duquesne University, USA

Jie Zhang, The College at Brockport State University of New York, USA

\section{${ }^{*}$ Correspondence:}

Stefan Billinger, School of Health and Medical Sciences, Örebro University, SE-701 82, Örebro, Sweden.

e-mail: stefan.billinger@telia.com
Students' capabilities to use symbolic information in classroom setting could be expected to influence their possibilities to be active and participating. The development of strategies for teachers to compensate for reduced capability need specific operational definition of symbolic behavior. Fifty-three students, aged 11-13 years old, 29 boys and 24 girls, from three classes in the same Swedish compulsory regular school participated in the current study. After a short training sequence 25 students (47\%) were defined as showing symbolic behavior (symbolic), and 28 students (53\%) were not (non-symbolic), based on their follow-up test performances. Symbolic and non-symbolic differed significantly on post-test performances $(p<0.05)$. Surprisingly, nonsymbolic behavior deteriorated their performance, while symbolic enhanced their performance $(p<0.05)$. The results indicate that the operational definition used in the present study may be useful in further studies relating the capability to show symbolic behavior and students' activity and participation in classroom settings.

Keywords: symbolic behavior, stimulus equivalence, matching-to-sample, classroom setting

\section{INTRODUCTION}

One central aspect of such complex situation as a regular classroom situation would probably be student's capacities to functionally use symbolic information (e.g., Bruce, 2007; Dockrell and Lindsay, 2008). Children's conversations at home may focus on here-andnow, people, action, and objects presenting in the immediate context. In school classroom, language is far more decontextualized - abstracted from the context in which it might be uttered. In school classroom, the focus of the conversation is mostly directed by the teacher and will be full of information about subjects that the student may have had no prior direct experience of. Previous research has documented a strong connection between teacherdirected instructions and student achievement and learning behavior in regular classroom settings (Gettinger and Stoiber, 2009). One way to look at it is that children need to learn to respond to decontextualized symbolic information (Hayes et al., 2001) very quickly in classroom in order to understand, be active, and participating. The development of the ability to use symbols is a central and fundamental ability for more complex human cognitive development and one of the keys for understanding human behavior (McCune, 2010).

Descriptions of children's decontextualized behavior are used in previous research (Morgan and Goldstein, 2004; Curenton et al., 2008; Marvin and Cline, 2010). A problem with descriptions of decontextualized behavior from an operational perspective is that it is imprecise (Wilkinson and McIlvane, 2001). It does not allow a specification of when a student shifts from the use of "non-symbolic" to "symbolic" behavior, i.e., when the student let go of context-dependent behavior and shift to complete contextual free behavior. Stimulus equivalence methodology may offer a more functional approach; a working model of symbolic functioning which may contribute to answers in both laboratory as well as naturalistic settings.
Sidman (1971) conducted the first experiment on the phenomenon known as stimulus equivalence in an attempt to devise more effective methods for teaching reading comprehension. Stimulus equivalence is a phenomenon in which untaught relationships emerge as a function of the teaching in matching-to-sample instructional procedures. The Sidman (1971) study involved a student with learning disabilities who had been trained to match spoken words to pictures and spoken words to printed words, and who subsequently matched printed words to pictures without additional training. Such indirectly trained or novel associations between stimuli are now by some referred to as derived stimulus relations, as opposed to those associations that have been explicitly trained (Hayes et al., 2001). Equivalence relations are in the present study used as a model of symbolic relations versus associative relations for non-symbolic relations. Attempts to link the stimulus equivalence phenomenon to explanations of new forms of untaught verbal behavior (e.g., novel associations) have led to several theories, of which the relational frame theory is perhaps the most promising, in terms of explaining, understanding, modifying, or improving such behaviors (BarnesHolmes et al., 2004b).

Methodology based on derived stimulus relations may add a measure with greater operational precision to observe shifts from non-symbolic behavior to symbolic behavior. Previous research has applied this methodology and shown that it is possible to use skill-building strategies to train the use of symbolic information in students with developmental disabilities (Barnes-Holmes et al., 2001, 2004a; Rehfeldt and Barnes-Holmes, 2009). Literature addressing the use of symbolic information, indicates its importance for human functioning in general. Accordingly students' capabilities to respond to symbolic information and to show symbolic behavior will probably make a difference in students' activity and participation in classroom instructions. Previous research has 
showed that students with developmental disabilities vary in their ability in using symbolic information. An overall question is in what way variations among students in regular classrooms to use symbolic information influence their activity and participation during class. Empirical studies addressing that question need more precise operation of when the students shift from non-symbolic to symbolic behavior.

The purpose of the current study was to apply the operational model of one aspect of symbolic functioning in regular classroom settings. To do so we employed a basic matching-tosample procedure commonly used to emerge stimulus equivalence (Sidman, 1994; Green and Saunders, 1998). Typically, matching-to-sample procedures have the purpose to establish conditional relations. A short description of the procedure is that it starts with the presentation of a sample stimulus and two or more comparison stimuli. One of the comparisons matches the sample stimulus and the others do not. In the following training trials, in order to establish conditional discriminations, a response to the correct comparison stimulus will be followed by a reinforcer. The basic logic behind the design used in the current study is to employ the same procedure independently three times in three different classrooms. If the same pattern appears in three different classrooms - in the same school, it is reasonable to believe that there is a causal relation between the training trails and the symbolic behavior. Based on previous research in stimulus equivalence, it was hypothesized that (a) some students were expected to show symbolic behavior and some students were not based on their scores on the post-test trials, and (b) there would be no significant difference between post-test and follow-up scores, for neither group of students (symbolic or non-symbolic), thereby indicating a consistency in non-symbolic or symbolic behavior.

\section{MATERIALS AND METHODS PARTICIPANTS}

A total of 53 students, aged 11-13 years old, 29 boys and 24 girls, recruited from three different classes, participated in the study. All three classes were from the same compulsory regular school in Sweden. The target school had approximately 200 students ranging in age from 6 to 13 years old and is regarded as a normal school without any particular differential characteristics and the three classes were also seen as "ordinary" school classes.

\section{MATERIALS}

Paper pad

Each student was given a paper pad at the beginning of each session in the experiment. The pad had a page for each training and test trial. Every second page in the pad was colored (to facilitate for the test leader to see when all students had made their response in that trial and had turned to the next page). Each page in the pad was numbered with the trial number in small numerals in the bottom right corner. Each page had three printed $\mathrm{X}$ crosses (X X X), representing the row of three comparison stimuli presented on the silver screen in front of the class. The children were instructed to circle the cross which represented the comparison stimulus they thought matched the sample stimulus.

\section{Visual stimuli}

Throughout the experiment, nine main visual stimuli were used (see Figure 1). To facilitate explanation, each stimulus will be designated with an alphanumeric label (e.g., A1, B2, C3), which were never shown to the students. The stimuli were images of familiar things, such as Swedish words for "dog" (A1), "cat" (A2), "rabbit" (A3), and colored patches (blue-patch, B1; red patch, B2; green-patch, B3), and finally geometric forms (rectangle, C1; rhombus, C2; trapezoid, C3). Six additional visual stimuli were used as comparison stimuli in the training trials but not in the test trials. The nine main stimuli that were used altogether as comparison stimuli in the nine test trials (post-tests and follow-up tests), had been used differently in the training trials.

In all matching-to-sample training and test trials, these visual stimuli were projected on a silver screen, visible to all students in the class at the same time. A projector was used for the visual projection of the stimuli from a PowerPoint file.

\section{DESIGN}

The purpose of this experiment was to exam the relation between the training sequence and students showing symbolic behavior. Altogether 42 training trials and 24 test trials were conducted in each class during the three sessions with matching-to-sample procedures. The first session was designed as a pre-test, training, post-test procedure. The second session was designed as a training-post-test procedure repeated across two conditions (training of two stimulus classes; see Figure 2). The basic logic behind the design was to be able to determine whether students would score as if they were capable of "seeing" the derived (indirect) equivalent stimulus relations, after a fixed number of training trials. Green and Saunders' (1998) "Minimal Training and Test Trial Types for a Prototypic Stimulus Equivalence Experiment" (p. 241) was used as the basic model for the training and test procedure used in the present study. This model was chosen due to its minimal design which was believed to increase the social acceptability for the procedure. The third session only consisted of follow-up post-tests of indirectly trained relations in all three stimulus classes.

A mixed two-way ANOVA was used to evaluate the effects of the short matching-to-sample training on students' test trial scores. The within-subject factor was training, that is the number of correct scores on the pre-test and the average number of correct scores from the three stimulus classes on post- and follow-up tests of indirectly trained stimulus relations. The between-subject factor was whether or not the student was defined as showing symbolic behavior. Finally, three ANOVAs were performed with the same within and between-subject factors and the same pre-test but only with post- and follow-up tests concerning every stimulus classes.

When a student had performed all 18 of 18 mixed training trials correctly $(100 \%)$, and at least 8 out of 9 of the follow-up test trials correctly ( $89 \%)$, he/she was considered to show a symbolic behavior. Rather than excluding students who probably saw the indirect stimulus relation but made some careless mistake at the scoring, we chose to use a marginal 8 out of 9 . However if a student who had $100 \%$ correct training trials (18 of 18), but performed less than $89 \%$ in the follow-up test trials (i.e., less than 8 out of 9 ), he/ she was considered as failing to show symbolic behavior. The probability is very small for a student to score 8 out of 9 trials correctly in a row, with three options at every trial, by chance. 


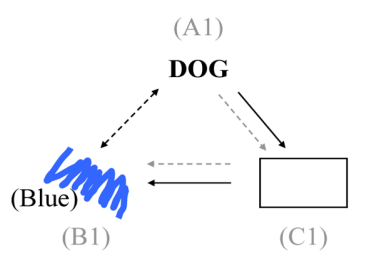

\section{Stimulus class 1.}

In stimulus class 1 , the relations of $\mathrm{A} 1 \mathrm{C} 1$ and $\mathrm{C} 1 \mathrm{~B} 1$ were trained in the first session.

The indirectly trained equivalent relation $\mathrm{A} 1 \mathrm{~B} 1$ was tested in both the A1B1 and the B1A1 direction in the first, the second and the follow-up sessions.
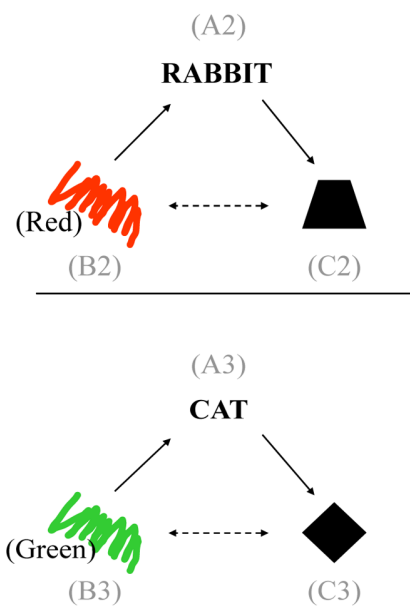

Stimulus class 2 .

In stimulus class 2, the relations of $\mathrm{B} 2 \mathrm{~A} 2$ and $\mathrm{A} 2 \mathrm{C} 2$ were trained in the second session.

The indirectly trained equivalent relation $\mathrm{B} 2 \mathrm{C} 2$ was tested in both the $\mathrm{B} 2 \mathrm{C} 2$ and the $\mathrm{C} 2 \mathrm{~B} 2$ direction in the second and the follow-up sessions.

\section{Stimulus class 3 .}

In stimulus class 3 , the relations of $\mathrm{B} 3 \mathrm{~A} 3$ and $\mathrm{A} 3 \mathrm{C} 3$ were trained in the second session.

The indirectly trained equivalent relation $\mathrm{B} 3 \mathrm{C} 3$ was tested in both the $\mathrm{B} 3 \mathrm{C} 3$ and the $\mathrm{C} 3 \mathrm{~B} 3$ direction in the second and the follow-up sessions.

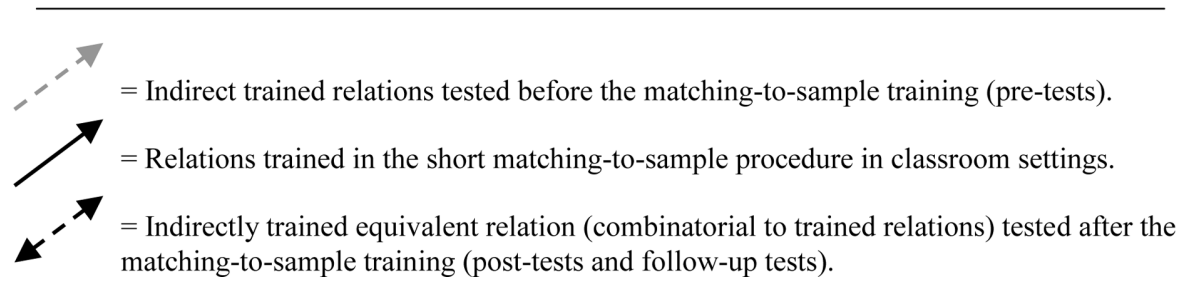

FIGURE 1 |The figure presents the three stimulus classes $(1,2$, and 3$)$ used in the current study, and the stimulus relations that were pre-tested, trained, post-tested, and tested in the follow-up session. The words (Blue), (Red), (Green), in the trial examples illustrated, were not visible to the students. The same applies to Figure 2.

\section{PROCEDURE}

All students participated of their own free will, and could leave the study at anytime. The students' parents were informed of the study by letter and had to give their written approval of their child's participation in the study. Initially 70 students participated in this study but eventually only 53 students followed all procedures and completed the study. Fifty-three of the initially 70 students scored all training trials correct at the end of training sequences, and they were on that ground included in the complete study. The present study was conducted consistent with the ethical rules and considerations within the national Swedish act "Ethical Review of Research Involving Humans (2003:460).” All three sessions in the study was conducted in the student's regular school class and regular classroom. The main structure of the procedure was the same in all three sessions. The students sat in their regular seats taking notes, and the experimenter stood in front of the class showing pictures. The procedure in the present study took about 10-20 min to administrate. Both the experimenter and the teacher were present in the classroom during the procedure encouraging the students not to whisper to each other, but to work independently.
The students were trained and tested in the formation of three 3-member equivalence classes (A1-B1-C1, A2-B2-C2, A3-B3C3; see Figure 1). Two of the relations between stimuli in each stimulus class were trained and one relation was tested during the experiment. The three stimulus classes that were to be trained and tested were randomly defined before the study started. The stimuli within each stimulus class are related to each other as same as. Which stimuli that would relate to each other as same as within each stimulus class, was randomly made up before the experiment. For example it is just by chance that the stimulus DOG was related as same as to the blue-patch and the rectangle and not the greenpatch and the trapezoid. In other words, all three arbitrary stimuli relations in each stimulus class (e.g., A1B1, A1C1, B1C1, i.e., DOGblue-patch, DOG-rectangle, blue-patch-rectangle) are random, and none of the students could possibly have had any prior knowledge of which stimuli would relate to each other as same as within each stimulus class.

All trials started such that the sample stimulus [for example the word DOG (A1)] was visible in the upper center part of the screen. After that, three comparison stimuli were visible in a row, 
in the lower part of the screen [for example a circle (C1), a triangle, and a rectangle]. The student's task was to choose one of the three comparison stimuli to match the sample stimulus. After every matching-to-sample training trial, the students were presented with information about which comparison stimulus was the right one in the trial. An arrow from the sample stimulus to the correct comparison stimulus appeared (see Figure 2) and a yellow circle appeared around the comparison stimulus that belonged to the same stimulus class as the presented sample stimulus, and the experimenter informed the students that the comparison stimulus belonged to the sample stimulus. After the matching-to-sample training trials, no such information was presented.

\section{RESULTS}

In the present study 53 students of the initially 70 students, scored all training trials correct at the end of training sequences. Of those 53 students, 28 (53\%, 15 boys, 13 girls) did not show symbolic behavior in the post-test trials (non-symbolic), while 25 (47\%, 14 boys, 11 girls) showed symbolic behavior in post-test trials (symbolic). These 53 students were sub grouped in to a non-symbolic student group and a symbolic student group.

A mixed two-way ANOVA was performed with Training (pretest, post-test, follow-up test) as the within-subject factor and Symbolic Behavior as the between-subject factor. The dependent variable was the number of correct scores on the pre-test, posttest, and follow-up test. Data analyses showed there was a significant impact of Training $[F(2,102)=66.56, p<0.001]$. Post hoc tests (Pair-Samples $t$-test, $5 \%$ level) showed higher scores in both post-test and follow-up when compared to the pre-test. That result indicates that the training had a significant impact on the students' capabilities to show symbolic behavior.

Step 1 in the first session. Three pre-test trials of indirectly trained stimulus relation. (C1B1, A1C1, C1B1) in stimulus class 1.

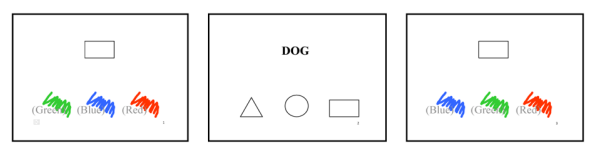

Step 2, the last part. Six training trials (mixed A1C1/C1B1) in stimulus class 1.
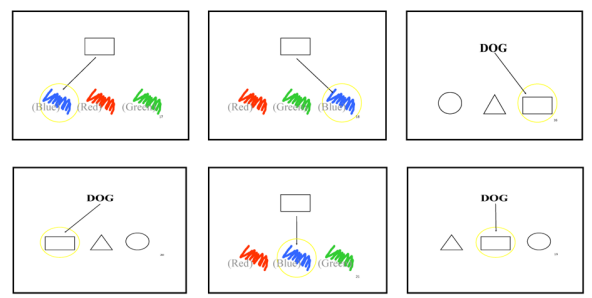

Step 3 in the first session. Three post-test trials (and follow-up trials in session three) of indirectly trained stimulus relations (B1A1, A1B1, B1AI) in stimulus class 1.

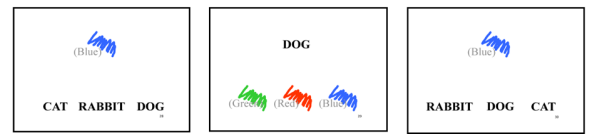

FIGURE 2 |Training and test trials in stimulus class 1 (all three steps in the first session).
There was also a significant impact on Symbolic Behavior [ $F(1$, $51)=22.90, p<0.001$ ]. Post hoc tests (Independent-Samples $t$-test, $5 \%$ level) showed no significant difference between non-symbolic and symbolic in the pre-test, but with regard to the post-test and the follow-up. The symbolic students scored significantly higher compared to the non-symbolic students. The result indicates that the training only had a significant impact on some of the students' capabilities to show symbolic behavior.

Finally, there was also a Training $\times$ Symbolic behavior interaction effect. Data analyses showed that the non-symbolic students first improved their scores on the post-test when compared to the pre-test, but then significantly lower their scores in the follow-up when compared to the post-test. The performance of symbolic students not only improved in the post-test when compared to the pre-test, but also significantly improved on the follow-up when compared to the post-test. For means and SD see Table 1. That result indicates that the training had a significant lasting impact on the symbolic students' capabilities to show symbolic behavior.

Subsequently similarly analyses (mixed two-way ANOVA, 5\% level) were performed where post-test and follow-up results for each stimulus class were compared with the pre-test. The analyses yielded the same significant indications in regard to Training, Symbolic Behavior, or interaction effects as already been reported for the average scores, with the exception of Stimulus class 3 where there was no difference between post-test and follow-up in the symbolic students. For means and SD see Table $\mathbf{1 .}$

Low scores on pre-test and high scores on post-test indicate that the students have learned to respond to (to "see") derived (indirect) stimulus relations within the stimulus class. The students' pre-test scores indicate that they had no previous experience of the stimulus relations before the short training sequence. Then, just a few

Table 1 | Mean values ( $M$ ) and standard deviations (SD) for scorings concerning the pre-test (bold), total post-test, total follow-up, and stimulus classes (1-3) during post-test, and follow-up in regard to symbolic behavior (non-symbolic versus symbolic).

\begin{tabular}{|c|c|c|c|c|c|c|}
\hline \multirow{2}{*}{ Test conditions } & \multicolumn{3}{|c|}{ Non-symbolic } & \multicolumn{3}{|c|}{ Symbolic } \\
\hline & $n$ & $M$ & SD & $n$ & $M$ & SD \\
\hline Pre-test & 28 & 0.96 & 0.92 & 25 & 0.92 & 1.12 \\
\hline Total post-test & 28 & $2.20 \mathrm{a}$ & 0.74 & 25 & $2.67 * a$ & 0.40 \\
\hline Total follow-up & 28 & $1.56 a$ & 64 & 25 & $2.89 * a$ & 0.16 \\
\hline Pre-test & 28 & 0.96 & 0.92 & 25 & 0.92 & 1.12 \\
\hline Stimulus class 1 post & 28 & $2.07 \mathrm{a}$ & 1.18 & 25 & $2.48 * a$ & 0.92 \\
\hline Stimulus class 1 follow & 28 & $1.75 a$ & 1.21 & 25 & $2.96 * a$ & 0.20 \\
\hline Pre-test & 28 & 0.96 & 0.92 & 25 & 0.92 & 1.12 \\
\hline Stimulus class 2 post & 28 & $2.21 \mathrm{a}$ & 1.13 & 25 & $2.92 * a$ & 0.40 \\
\hline Stimulus class 2 follow & 28 & $1.68 \mathrm{a}$ & 1.02 & 25 & $2.84 *$ & 0.37 \\
\hline Pre-test & 28 & 0.96 & 0.92 & 25 & 0.92 & 1.12 \\
\hline Stimulus class 3 post & 28 & $2.32 \mathrm{a}$ & 0.98 & 25 & $2.60 * a$ & 0.71 \\
\hline Stimulus class 3 follow & 28 & $1.21 a$ & 1.23 & 25 & $2.88 * a$ & 0.73 \\
\hline
\end{tabular}

"*" Indicates when students with symbolic behavior scored significantly higher when comparing to students with non-symbolic behavior.

" $\mathrm{a}$ " Indicates a significant difference from the tabulated value above. 
minutes later at the post-test trials they response as they can see them. That response, acting on derived (indirectly learned) stimulus relations in the present study, was defined as symbolic behavior. High scores on both post-test and follow-up test indicated that was not just something accidental. The symbolic students learn to see the derived stimulus relation, and score on follow-up test as if they learn to see the indirect stimulus relations even better, whereas the scores from the non-symbolic students seem to indicate less ability to see the indirect stimulus relation. The scores indicated that the same score pattern repeat across all three stimulus classes. It also indicated that some students seem to have an ability to quickly "see" the indirect stimulus relations and show symbolic behavior. The overall results indicate that the procedure used in the current study have a potential to differentiate between directly learned student behaviors and indirectly symbolic students' behaviors in classroom settings.

\section{DISCUSSION}

The present study had two hypotheses: (a) Some students were expected to show symbolic behavior and some students were not, based on their scores on the post-test trials, and (b) there would be no significant differences between post-test and follow-up scores, for neither group, thereby indicating a consistency in non-symbolic or symbolic behavior.

As hypothesized, some students in the study showed their capability to relatively easily use symbolic information and show symbolic behavior, after only short training in regular classroom settings. This result indicate that an operational definition of symbolic behavior based on stimulus equivalence methodology, as suggested by Wilkinson and McIlvane (2001), may be use to distinguish between behavior that is guided by non-symbolic relations (associative relations) and symbolic relations in natural settings. The possibility to observe more precise variations in showed symbolic behavior, as a complement to description of decontextualized behavior, allows for more precise empirical testing of hypotheses in specific contexts. The present results focused on classroom settings, confirm previous research in showing that students' capabilities of using symbolic information vary (Barnes-Holmes et al., 2001, 2004a; Rehfeldt and BarnesHolmes, 2009). However as far as we know, no previous research has found solid data indicating variations among students' capabilities to show symbolic behavior in regular classroom setting after short training.

The theoretical assumption behind the hypothesis is that if a group of students score all correct responses on the test trails after the training session, they have previously learned to more easily and automatically respond to indirect (symbolic) relation from directly trained or experienced relations (Hayes et al., 2001). The training trials are supposed to give necessary and sufficient information for a majority of the students to respond to the symbolic relations, which are the untaught, indirect trained relations. The assumption is that this might be an analog between the short training sequence used in the present study and a short teacher instruction. This might reflect an aspect of what might happen every day in regular classrooms, some students have the ability to "see" and act upon symbolic or indirect relations within the systematic information given by the teacher. While other students might not have that ability, which might be a functional aspect that holds some students back in instructional situations, for example, some students with lower cognitive abilities.

Surprisingly, results from our study did not support the second hypotheses. Results indicated that the difference between the students who showed symbolic behavior and those who did not, were more evident in the follow-up data a few days after training. The children with no symbolic behavior deteriorated their performance, while children with symbolic behavior enhanced their performance. This indicates that the short training sequence seemed to have made a profound and long lasting impact on the students. Difference in how they would respond and behave in situations was not directly associated to the short training sequence. The group of students, who failed to score correctly on follow-up test trials but did not differ (from the group of students who showed symbolic behavior) on pre-test or training trials, were defined in the current study as failing to show symbolic behavior. Within a more functional theoretical framework, it means these students failed because they had not learned to automatically respond to the symbolic relation under these premises. Their symbolic behavior has not been reinforced enough to the point where the perception of the symbolic relation automatically would guide their response (Luciano et al., 2007). This functional hypothesis is yet to be empirical tested by further studies. An alternative way to address why these students did not score correctly particularly in post- and follow-up test trials, although they did not differ from pre-test and training trails, might be to use more concepts on another level, such as a lack of motivation or lower intellectual abilities. No previous research has found existing data indicating that variations among students' capabilities to show symbolic behavior in a regular classroom setting after short training instructions.

The basic idea in the present study was to test whether students would response to stimulus relations they did not had any previous direct systematic reinforcing experience of. But, could there be any unaware systematic direct reinforcement during the training trails that makes the test trials directly trained stimulus relations rather than derived symbolic relations? The nine main stimuli that were used altogether as comparison stimuli in the nine test trials (post-tests and follow-up tests), had been used differently in the training trials.

The stimulus DOG (A1) had neither appeared as a "correct" nor "incorrect" comparison stimulus in any trial during the training. The stimulus RABBIT (A2) had appeared as "correct" in 6 trials, but never as "incorrect." The stimulus CAT (A3) had appeared as "correct" in 6 trials, and as "incorrect" in 6 trials. The stimulus "blue-patch" (B1) had appeared as "correct" in 9 trials, but never as "incorrect." The stimuli "red patch" (B2) and "green-patch" (B3), had both only appeared as "incorrect" in 9 trials. The stimulus "rectangle" (C1) had appeared as "correct" in 9 trials but never as "incorrect." The stimulus "rhombus" (C2), had appeared as "correct" in 3 trials but never as "incorrect," and the stimulus "trapezoid" (C3) had appeared as "correct" in 3 trials, and as "incorrect" in 3 trials. So, if the student strictly responded according to which comparison stimulus that had been associated with most positive reinforcement during the training, the student would get 1 test 
trial correct out of 9 altogether. So neither extraneous rules nor extraneous direct reinforcement could easily be seen as alterative explanation to why a majority of the students scored all test trails correct.

The present results indicated that the short training sequence was sufficient to give "the symbolic students" experiences that guide their behavior even more easily days after the training, whiles the opposite seemed true for "the non-symbolic students." The short training sequence seemed to have made a profound and long lasting impact on the students. But from the results of our current study, we cannot confirm yet whether or not the observed symbolic behavior is analog to meaningful student behavior manifesting from the use of symbolic information in classroom settings. To be able to predict more precisely when students may succeed or fail to be active and participate in classroom settings after short teacher instructions, further studies are needed.

\section{REFERENCES}

Barnes-Holmes, D., Barnes-Holmes, Y., and Cullinan, V. (2001). "Education," in Relational Frame Theory. A PostSkinnerian Account of Human Language and Cognition, eds S. C. Hayes, D. Barnes-Holmes, and B. Roche (New York: Kluwer Academic/ Plenum Publishers), 119-139.

Barnes-Holmes, D., Barnes-Holmes, Y., and Murphy, C. (2004a). "Teaching the generic skills of language and cognition: contributions from relational frame theory," in Evidence-Based Educational Methods, eds J. M. Moran and R. W. Malott (San Diego: Elsevier Academic Press), 277-293.

Barnes-Holmes, D., Barnes-Holmes, Y., Smeets, P. M., Cullinan, V., and Leader, G. (2004b). Relational frame theory and stimulus equivalence: conceptual and procedural issues. Int. J. Psychol. Psychol. Ther. 4, 181-214.

Bruce, B. (2007). Problems of Language and Communication in Children, Identification and Intervention. Doctoral thesis, Department of Logopedics, Phoniatrics and Audiology, Lund University.

Curenton, S. M., Craig, M. J., and Flanigan, N. (2008). Use of decontextualized talk across story contexts: how oral storytelling and emergent reading can scaffold children's development. Early Educ. Dev. 19, 161-187.

Dockrell, J. E., and Lindsay, G. (2008) "Inclusion versus specialist provision for children with development language disorders," in Understanding Developmental Language Disorders, eds C. F. Norbury, J. B. Tomblin, and V. M. Bishop (New York: Psychology Press), 131-147.

Gettinger, M., and Stoiber, K. (2009). "Effective teaching and effective schools," in The Handbook of School Psychology, 4th Edn, eds T. B. Gutkin and C.R. Reynolds (New York: Wiley), 769-790.

Green, G., and Saunders, R. R. (1998). "Stimulus equivalence," in Handbook of Research Methods in Human Operant Behavior, eds K. A. Lattal and M. Perone (New York: Plenum Press), 229-262.

Hayes, S. C., Fox, E., Gifford, E. V., Wilson, K. G., Barnes-Holmes, D., and Healy, O. (2001). "Derived relational responding as learned behavior," in Relational Frame Theory. A Post-Skinnerian Account of Human Language and Cognition, eds S. C. Hayes, D. Barnes-Holmes, and B. Roche (New York: Kluwer Academicl Plenum Publishers), 21-50.
Our main conclusion is that the procedure used in the current study has a potential to differentiate between directly learned student behaviors and indirectly symbolic students' behaviors in classroom settings. Although further work is required to gain better understanding on how to present information in classroom more effectively so that all students have the equal chance to respond to symbolic information, our findings indicated an opening for more empirical research on symbolic behavior in natural settings such as in regular classrooms. That might help us further find out on a functional level which student might be at risk in different instructional situations.

\section{AUTHOR'S NOTES}

The research plan has followed the ethical standards of the Swedish act "Ethical Review of Research Involving Humans" and has been accepted by the board of the Ethical Committee of Örebro University.

Luciano, M. C., Gómez-Becreea, I., and Rodriguez-Valverde, M. (2007). The role of multiple-exemplar training and naming in establishing derived equivalence in an infant. J. Exp. Anal. Behav. 87, 349-365.

Marvin, C. A., and Cline, K. D. (2010). Bus talk: a preliminary analysis of children's decontextualized talk. Commun Disord. Q. 31, 170-182.

McCune, L. (2010). "Developing symbolic abilities," in Symbolic Transformation: The Mind in Movement Through Culture and Society, ed. B. Wagoner (London: Taylor \& Francis), 193-208.

Morgan, L., and Goldstein, H. (2004). Teaching mothers of low socioeconomic status to use decontextualized language during storybook reading. $J$. Early Interv. 26, 235-252.

Rehfeldt, R. A., and Barnes-Holmes, Y. (2009). Derived Relational Responding: Applications for Learners with Autism and Other Developmental Disabilities. Oakland, CA: New Harbringer.

Sidman, M. (1971). Reading and auditoryvisual equivalence. J. Speech Hear. Res. 14, 5-13.

Sidman, M. (1994). Equivalence Relations and Behavior: A Research Story. Boston: Authors Cooperative.
Wilkinson, K. M., and McIlvane, W. J. (2001). Methods for studying symbolic behavior and category formation: contributions of stimulus equivalence research. Dev. Rev. 21, 355-374.

Conflict of Interest Statement: The authors declare that the research was conducted in the absence of any commercial or financial relationships that could be construed as a potential conflict of interest

Received: 04 November 2010; accepted: 28 May 2011; published online: 13 June 2011. Citation: Billinger $S$ and Norlander $T$ (2011) Symbolic behavior in regular classrooms: a specification of symbolic and nonsymbolic behavior. Front. Psychology 2:122. doi: 10.3389/fpsyg.2011.00122

This article was submitted to Frontiers in Educational Psychology, a specialty of Frontiers in Psychology.

Copyright $(2011$ Billinger and Norlander. This is an open-access article subject to a non-exclusive license between the authors and Frontiers Media SA, which permits use, distribution and reproduction in other forums, provided the original authors and source are credited and other Frontiers conditions are complied with. 